\title{
Weitere Mittheilungen über die intraperitoneale Infection der Meerschweinchen mit Cholerabakterien. ${ }^{1}$ Von

\author{
Dr. med. O. Voges
} \\ in Danzig.
}

In unserer ersten Mittheilung über die intraperitoneale Chölerainfection der Meerschweinchen hatten wir den Nachweis erbracht, dass es sich bei derselben nicht um denselben Vorgang handelte, wie bei der Enzyminfection. Die folgenden Zeilen sollen dazu dienen, weitere über dieses Thema angestellte Untersuchungen zu schildern. In seinen Untersuchungen über das Choleragift kommt Pfeiffer $^{2}$ zu folgenden Ergebnissen :

„In ganz jungen, aërob gezüchteten Choleraculturen ist ein specifischer Giftstoff enthalten, welcher ausserordentlich intensive toxische Effecte entfaltet. Dieses primäre Choleragift steht in sehr enger Zusammengehörigkeit zu den Bakterienleibern und ist vielleicht ein integrirender Bestandtheil derselben. Durch Chloroform, Thymol und durch Trocknen können die Choleravibrionen abgetödtet werden, ohne dass dieser Giftstoff anscheinend verändert wird. Alcohol absolutus, concentrirte Lősungen der Neutralsalze, Siedehitze zersetzen ihn und lassen secundäre Giftkörper zurück, die eine ähnliche physiologische Wirkung haben, aber erst in der 10 bis 20 fachen Dosis den gleichen toxischen Effect erzielen. Auch die anderen Mitglieder der Vibrionenfamilie - der Vibrio Metschnikoff und der Finkler'sche Kommabacillus - enthalten nahe verwandte Giftstoffe."

\footnotetext{
1 Eingegangen am 12. April 1894.

Diese Zeitschrift. Bd. XI. S. 411 ,
} 
Diese Mittheilungen lassen somit vermuthen, dass wir den Giftstoff in den Zellleibern zu suchen haben, und fragt es sich, ob dieses auch für die anderen Bakterien, die Meerschweinchen nach intraperitonealer Infection unter ähnlichen Symptomen zu tödten vermögen, der Fall ist.

Von dem Filtrat einer 24stündigen Heubacillusbouilloncultur werden einem Meerschweinchen von $490 \mathrm{grm}$ Gewicht $4 \mathrm{com}$ intraperitoneal injicirt, ohne dass das Thier irgend welche Reaction erkennen liess. Der nämliche Versuch wird mit dem Micrococcus prodigiosus mit demselben Ausgang angestellt.

Diese Versuche lassen somit schliessen, dass es sich ebenfalls um ein in der Zellsubstanz eingeschlossenes Gift handeln musste, ähnlich wie beim Choleravibrio, und fragt es sich nur, ob dieser Giftstoff durch eine allmähliche Auslaugung - vielleicht eine Art Diffusion - oder durch den Zerfall der Zelle frei gemacht wird.

Buchner ${ }^{1}$ berichtet über Versuche, in denen er durch ein bestimmtes Verfahren die von ihm als Proteïne bezeichneten Stoffe aus dem Bakterienleibe darstellen konnte. Wir versuchten diese Methode für unsere Versuche anzuwenden. Die von Agar-Agarmassenculturen abgekratzten Bakterienmengen wurden im Trockenschranke bei $100^{\circ} \mathrm{C}$. getrocknet und das Pulver, in Wasser aufgeschwemmt, 4 Wochen lang täglich 2 Stunden im Wasserbad gekocht. Alsdann wurde die Masse durch ein Pukall'sches Filter gejagt. Die mit dem Filtrat angestellten chemischen Untersuchungen liessen die Xanthoproteïn, Biuret und Millon'sche Reaction erkennen. Als wir jedoch den peptonfreien Uschinsky'schen Nährboden - auf dem der von uns alsdann verwandte Micrococcus prodigiosus sehr gut fortkommt - benutzten, fielen alle diese Reactionen fort, so dass der frühere positive Ausfall nur auf Kosten des mit den Culturen abgekratzten Peptons zu setzen ist, eine Fehlerquelle, die man durch Benutzung peptonfreier Nährböden vermeiden kann.

Von dem Filtrat der ausgekochten Heubacillenculturen wurden an Meerschweinchen gewisse, nicht näher definirbare Mengen intraperitoneal injicirt. Andere Thiere erhielten die nämliche Dosis subcutan. Die Giftwirkung setzte fast momentan ein. Grosse Dosen bewirkten einen bis auf $28^{\circ} \mathrm{C}$. und weniger herabsteigenden Temperatursturz; bei Anwendung geringerer Mengen trat jedoch eine Temperatursteigerung um 1 bis $2^{0} \mathrm{C}$. ein. Die subcutan geimpften Thiere reagirten in gleicher Weise, bekamen jedoch nie Hautgeschwüre, eine Thatsache, die in der schnelleren Resorbirbarkeit des aufgelösten Giftes begründet sein kann. Die in der nämlichen Weise wie vom Bacillus subtilis hergestellten Filtrate vom Micrococcus

. Münchener medicinische Wọchensehrift. 1891. Nr. 49, 
prodigiosus wurden in der gleichen Weise an Thieren erprobt und ergaben genau dasselbe Resultat. Temperatursteigerung um 1 bis $2^{\circ} \mathrm{C}$. warde beobachtet nach der Injection kleinerer Mengen, Temperatursturz entweder mit nachfolgendem Exitus oder Rückkehr zur Norm ohne nachfolgende Steigerung nach Injection grosser Dosen. Auf mehrfach hintereinander folgende Dosen reagirten die Thiere jedes Mal mit einer Temperatursteigerung. Es zeigte sich dabei noch die eigenthümliche Erscheinung, dass das Gewicht der Thiere ganz enorm abnahm, ein äusserster Marasmus führte schliesslich zum letalen Exitus. Wir hatten demgemäss eine Substenz vor uns, durch welche wir ungemein schädigend auf den Meerschweinorganismus einzuwirken vermochten. Welcher Art war nun diese Substanz und wie sollten wir sie noch reiner darstellen?

Dass es sich nicht um ein toxisch wirkendes Pepton bandelte, geht daraus hervor, dass aus peptonfreien Nährböden hergestellte Filtrate keine Peptonreactionen geben. Es musste sich um eine andere Substanz handeln, welche zweeks genauerer Untersuchung noch reiner dargestellt werden musste. Zwei Möglichkeiten boten sioh uns dar. Auf ähnliche Weise wie Brieger und $\mathrm{Cohn}^{1}$ das Tetanusgift darstellten, gingen wir vor. Das zur Syrupconsistenz eingeengte Filtrat wurde im Ueberschuss mit 30 Procent Ammonsulfatlösung versetzt, der nun entstandene Niederschlag durch die Centrifuge gesammelt, getrocknet und gepulvert. Das Pulver wurde mit reinem Chloroform ausgeschüttelt, woranf in das letztere Substanzen übergingen, welche dasselbe trübten und sich allmählich in den oberen Partieen ansammelten. Das Chloroform wurde abgehoben und völlig verdampft. Der Rückstand im Vergleich zur ursprünglichen Menge, eine äusserst geringe, graue, pulverförmige, amorphe Masse, löste sich in Wasser. Nach intraperitonealer Injection dieser in Wasser gelösten Masse in die Bauchhöhle eines Meerschweinchens schnellte dessen Temperatur von $37^{\circ} \mathrm{C}$. in $2^{1 / 2}$ Stunden bis auf $39^{\circ} \mathrm{C}$., um dann allmählich zur Norm zurückzukehren. Wir vermochten somit mit unserer Substanz genau die gleiche Temperatursteigerung hervorzubringen, wie mit dem Filtrat und durften hoffen, die Giftsubstanz in reinerer Form vor uns zu haben. Es war jedoch die Ausbente an wirksamen Stoffen eine so äusserst minimale, dass es fast unmöglich schien, auf diesem Wege auch nur.eine für einige Thiere hinreichende Giftmenge zu erzielen. Wir hatten nun die Beobachtung gemacht, dass durch einen grossen Ueberschuss Alcohol absol. ebenfalls und zwar ein viel voluminöserer Niederschlag erfolgte. Der Alkoholniederschlag wurde durch die Centrifuge gesammelt, getrocknet, gepulvert und mit Chloroform ausgeschüttelt. Das letztere trübte sich,

\footnotetext{
1 Diese Zeitschrift. Bd. XV.
} 
wurde abgehoben und verdunstet. Es restirte ein graues, amorphes Pulver, welches sehr leicht in Wasser löslich war, keine Eiweiss- und Peptonreaction darbot. Eine nicht näher bestimmte Dosis wurde einem Meerschweinchen injicirt und stieg die Temperatur in 2 Stunden um $1{ }^{1} /{ }^{\circ} \mathrm{C}$., um dann allmählich zur Norm zurückzukehren. Das Krankheitsbild war das nämliche wie bei dem mit dem Ammonsulfatniederschlag geimpften Thiere. - Die Thiere sind Anfangs etwas aufgeregt, da die Giftwirkung wohl sofort nach der Injection eintritt, dann kauern sie still in ihrem Käfig, ohne zu fressen, haben struppiges Haar, halbgeschlossene Augen und fibrilläres Zucken über dem ganzen Körper. Koth wird meist reichlichst entleert. Bei Berührung des Bauches, der mässig gespannt ist, klagen die Thiere laut. Anfangs macht sich ein geringes Heruntergehen der Temperatur bemerkbar, dann steigt dieselbe; wenn sie wieder zur Norm abfällt, fressen die Thiere allmählich wieder und erholen sich, doch bleibt eine Schwäche, wie nach Ueberstehen einer lang fieberhaften Krankheit längere Zeit bestehen. Durch das Ueberstehen dieser Intoxication verlieren die Thiere sehr an Gewicht, so dass der Gewichtsverlust bis $201 / 6$ des Gesammtkörpergewichtes betragen kann. Es dauert stets einige Zeit, bis dass das vor der Krankheit vorhanden gewesene Gewicht wieder hergestellt ist. Wenn die Thiere auch durch eine 24 stündige Inanition um $20 \mathrm{grm}$ abnehmen können, so können doch derartige Gewichtsverminderungen, wie die von mir beobachteten, nicht allein durch die Inanition bedingt sein. Wiederholen wir die Injection in so kurzen Intervallen, dass das Thier nicht wieder sein früheres Gewicht erlangt, so muss schliesslich ein Zustand erreicht werden, der die höchsten Grade von Marasmus ausdrückt. Es zeigt sich bei den nachfolgenden wie bei der ersten Impfung stets das nämliche Bild; die Thiere machen dieselben Temperaturschwankungen durch wie das erste Mal und wir haben es in der Hand, sie durch Injection grösserer oder kleinerer Dosen in beliebiger Weise zu verändern.

Soweit waren unsere Untersuchungen gediehen, als uns die Arbeit Centanni's ",Untersuchungen über das Infectionsfieber" zu Händen kam. Die von ihm aus einer ganzen Reihe pathogener wie nicht pathogener Bakterien isolirte und von ihm als Pyrotoxina bacterica benannte Substanz muss ich als die nāmliche, wie die von mir aus den Culturen des Bacillus subtilis und Micrococcus prodigiosus gewonnene bezeichnen. Die über die chemischen Eigenschaften dieser Substanz angegebenen Mittheilungen konnten wir ebenfalls grösstentheils bestätigen und machten ferner die Beobachtung, dass dieselbe auch im Stande ist, die Gelatine

1 Deutsche medicinische Wochenschrift. 1894. Nr. 7. u. 8. 
zu verflüssigen, so dass wir in ihr wohl ein proteolytisches Ferment vor uns haben. Diese Substanz ist jedoch absolut nichts Neues, da Buchner sie schon kannte, wenn auch unter anderem Namen, und auch Hueppe ${ }^{1}$ scheint mit ähnlichen Substanzen schon 1887 in seiner Arbeit gearbeitet zu haben, obwohl er uns über die angewandten Bakterienstoffe ziemlich im Unklaren lässt.

Issa eff ${ }^{2}$ berichtet über Untersuchungen über die künstliche Immunität gegen Cholera. Es lehrt uns diese Arbeit, wie wir bei der Beurtheilung der Versuche und Versuchsresultate die grösste Vorsicht anwenden sollten; so gelang es dem Autor durch intraperitoneale Injection der mannigfachsten Flüssigkeiten eine Immunität gegen eine nachfolgende Cholerainjection hervorzurufen. Da wir als Vehikel für die Injection immer sterilisirtes Wasser benutzten, fragte es sich, ob nicht dieses bereits einen Einfluss auf den Organismus der Meerschweinchen auszuüben im Stande sei.

Meerschweinchen $235^{\mathrm{grm}}$ schwer erhält $1^{\mathrm{ccm}} \mathrm{H}_{2} \mathrm{O}$ intraperitoneal.

Temperatur: $\quad 9^{\mathrm{h}} \quad 9^{1} /_{2^{\mathrm{h}}} \quad 10^{\mathrm{h}} \quad 11^{\mathrm{h}} \quad 12^{1} / 2^{\mathrm{h}} \quad 2^{\mathrm{h}} \quad 3^{\mathrm{h}} \quad 4^{\mathrm{h}}$ $\begin{array}{llllllll}38.3 & 38.8 & 38.8 & 40.1 & 39.3 & 38.8 & 38.7 & 38.3\end{array}$

Das Thier war äusserlich andauernd gesund. Mehrère andere Thiere zeigten eine ähnliche Curve.

Wenn Issaeff nachweisen konnte, dass die intraperitoneale Injection eine starke Leukocythenansammlung zur Folge hatte, so lehren diese Versuche, dass dieselbe mit einer nicht unbedeutenden Temperatursteigerung verbunden ist; da wir nun unsere Giftsubstanz in 1 com $\mathrm{H}_{2} \mathrm{O}$ pro Thier aufgeschwemmt hatten, so war es immerhin denkbar, dass die Fieberreaction nur durch das Wasser bedingt war.

Um diese Fehlerquelle auszuschliessen, injicirten wir einem Meerschweinchen von $225^{\mathrm{grm}} 1^{\mathrm{cem}} \mathrm{H}_{2} \mathrm{O}$ subcutan in die Nackengegend.

Temperatur: $\quad 9^{\mathrm{h}} \quad 9^{1} / 2^{\mathrm{h}} \quad 10^{\mathrm{h}} \quad 11^{1} /^{\mathrm{h}} \quad 12^{1} /^{\mathrm{h}} \quad 2^{\mathrm{h}} \quad 3^{\mathrm{h}} \quad 4^{\mathrm{h}}$ $\begin{array}{llllllll}38.6 & 38.6 & 38.5 & 38.5 & 38.6 & 38.3 & 38.4 & 38.4\end{array}$

Das Thier war andauernd gesund und zeigte auch keine Temperatursteigerung, dieser Versuch wurde mit demselben Resultat wiederholt. Injicirten wir dagegen $2^{\mathrm{cm}} \mathrm{H}_{2} \mathrm{O}$ subcutan, so trat auch dann eine Fieberreaction ein.

Temperatur beim Meerschweinchen ron $225^{\mathrm{grm}}$ :

$$
\begin{array}{cccccc}
4^{\mathrm{h}} & 5^{\mathrm{h}} & 6^{\mathrm{h}} & 7^{\mathrm{h}} & 8^{\mathrm{h}} & 10^{\mathrm{h}} \\
37.6 & 37.8 & 39.1 & 38.7 & 38.5 & 38 \cdot 1 .
\end{array}
$$

Diese Versuche lassen zur Genüge erkennen, wie ausserordentlich leicht die Thiere durch eine Wärmeschwankung auf den Eingriff einer

1 A. a. 0.

${ }^{2}$ Diese Zeitschrift. Bd. XVI. 
Wittere MittheiLUngen ÜBer INTRAPERItoneaLe INFECTION. 479

intraperitonealen oder subcutanen Injection reagiren. Wir benutzten daher für die Folge stets die Subcutaninjection wo es anging und wählten möglichst kleine Flüssigkeitsmengen, etwa 0.1 bis $0.2^{\mathrm{ecm}}$ Wasser pro Thier, um so diese Fehlerquelle nach Möglichkeit zu vermeiden. Dass es sich übrigens bei der Injection unserer Bakterienproducte um ein wirklich wirksames Princip handelt, geht schon daraus hervor, dass die mit Wasser behandelten 'Thiere äusserlich völlig gesund waren, während die mit dem Bakterientoxin behandelten doch mancherlei Krankheitssymptome darboten. Weit heftiger als das Wasser wirkte die 30 procentige Ammonsulfatlösung. $1 \mathrm{ccm}$ derselben subcutan injicirt, tödtete ein Meerschweinchen von $240 \mathrm{grm}$ innerhalb weniger Minuten. Da es immerhin möglich war, dass auch kleinere Dosen Ammonsulfat in das Chloroform übergingen, so zogen wir es vor, lieber die Alkoholniederschläge zu benutzen.

Das Toxin, welches wir durch den langwierigen Process des Kochens u. s. w. erhalten hatten, konnten wir übrigens auch aus alten Culturen direct gewinnen. Eine am 16./XII. 93 von $800^{\mathrm{ccm}}$ Uschinskilösung angesetzte Cholerácultur, welche aus einer unlängst aus dem Darm gezüchteten Choleracultur stammte, zeigte, nachdem sie in der Zwischenzeit meist im Thermostaten, sonst bei Zimmertemperatur gestanden, nach 100 Tagen noch reichliche Mengen lebender Cholerabacillen in Reincultur, welche auf Pepton gebracht, eine ausserordentlich intensive Indolbildung zeigten und für Thiere in der gleichen Dosis pathogen waren, wie frisch fortgezüchtete Culturen. ${ }^{1}$ Aus dem Filtrat dieser Uschinskiculturen wurde durch Alkohol ein ebenfalls wirksames Toxin ausgefällt, welches keine Millon'sche Xanthoprotheìn- und Biuretreaction gab. Das Thier zeigte die nämlichen Symptome wie die mit dem aus frischen Culturen gewonnenen Toxin inficirten Thiere.

Die Curve war folgende:

$$
\begin{array}{lclllllll}
10^{\mathrm{h}} & 10^{1} / /^{\mathrm{h}} & 11^{\mathrm{h}} & 12^{\mathrm{h}} & 1^{\mathrm{h}} & 2^{\mathrm{h}} & 3^{\mathrm{h}} & 4^{\mathrm{h}} & 5^{\mathrm{h}} \\
37 \cdot 6 & 38 \cdot 0 & 38.2 & 39.8 & 40.0 & 39.6 & 39 \cdot 1 & 38 \cdot 5 & 38 \cdot 3 .
\end{array}
$$

Es ist damit bewiesen, dass das Trocknen und Kochen der Bakterienmassen diese Substanz nicht wesentlich verändert, oder dass wenigstens durch diese Behandlung keine andere Veränderung hervorgerufen wird, wie durch das Altern der Culturen.

Von grösstem Interesse musste es sein, zu ermitteln, ob dieses Toxin das gegen die Cholerabacillen immunisirende Princip sei, und ob wir in

1 Noch bei der Durchsicht der Correctur liess sich aus dem Rest dieser Culturen eine Reincultur von Cholerabacillen züchten, so dass also der Cholerabacillns in dem völlig eiweiss- und peptonfreien Nährboden sicher 144 Tage lebens- und entwickelungsfähig ist. 
dieser Substanz das Choleragift vor uns haben. Centanni sagt in seiner Arbeit: „Der grösste Theil der von verschiedenen Autoren gewissen Bakterienarten zugeschriebenen Gifte lässt sich auf das Pyrotoxin zurūckführen; das angenommene specifische Gift ist entweder nicht vorhanden, oder es gelingt nicht, seine Bildung in unseren künstlichen Culturmitteln hervorzurufen.- So ist das Choleragift in unseren Culturen nichts als gewöhnliches Pyrotoxin."

Pfeiffer ${ }^{1}$ vertritt in seiner jüngsten Publication seine schon früher ausgesprochene Ansicht, dass in den Leibern der Choleravibrionen Giftsubstanzen enthalten sind, welche, in den gewöhnlichen Culturmedien fast unlöslich, im Körper der als Versuchsthiere benutzten Meerschweinchen nach dem Zugrundegehen der injicirten Bakterien frei werden und dann auf die Centren der Circulation und Temperaturregulirung lähmend wirken. Diese Giftstoffe sind in ungewöhnlichem Grade labil. Nach ihrer Zerstörung durch thermische oder chemische Eingriffe bleiben secundäre Giftkörper zurück, die in ihrer physiologischen Wirkung den primären Toxinen sehr ähnlich sich verhalten, aber erst in vielfach höherer Dosis denselben toxischen Effect hervorzurufen vermögen. Diese secundären Toxine sind relativ sehr resistente Substanzen, die sogar stundenlanges Kochen vertragen.

Sollen wir Centanni Recht geben, so muss es gelingen, erstens den Tod eines Meerschweinchens durch viel kleinere Dosen Pyrotoxin herbeizuführen wie durch die Injection einer Bakterienmasse, sodann müssen wir im Stande sein, Thiere durch Pyrotoxin gegen Cholera zu immunisiren.

Pfeiffer giebt in seiner obigen Abhandlung an, dass $2 \frac{1}{2}$ bis $5^{\mathrm{mgr}}$ pro $100^{\mathrm{grm}}$ Thier einer durch Chloroform sterilisirten Agarcultur von Cholerabacillen genügten, um den Tod eines Thieres herbeizuführen. Wir verwendeten dus Pyrotoxin des Prodigiosus, welcher nach Klein für Meerschweinchen mindestens in gleichem Grade pathogen ist, wie die Cholerabacillen. Von dem genau nach den Angaben Centanni's von den Uschinskiagarculturen hergestellte Pyrotoxin wurde in einer Dosis von $0 \cdot 3^{\mathrm{grm}}$ einem Meerschweinchen : von $250^{\mathrm{grm}}=0.12^{\mathrm{grm}}$ pro $100^{\mathrm{grm}}$ Thier injicirt.

Die Temperatureurve war folgende:

$$
\begin{array}{cccccccccc}
4^{1} / 2^{\mathrm{h}} & 5^{\mathrm{h}} & 6^{\mathrm{h}} & 7^{\mathrm{h}} & 8^{\mathrm{h}} & 9^{\mathrm{h}} & 10^{\mathrm{h}} & 12^{\mathrm{h}} & 4^{\mathrm{h}} & 6^{\mathrm{h}} \\
38 \cdot 4 & 36 \cdot 3 & \mathbf{3 4} \cdot 4 & 32 \cdot 0 & 34 \cdot 0 & \mathbf{3 5 \cdot 4} & \mathbf{3 7 \cdot 5} & 38 \cdot 1 & \mathbf{3 8} \cdot 4 & 38 \cdot 3
\end{array}
$$

Das Thier war nach der Injection still und traurig, hatte struppiges Fell, frass nicht und klagte leise bei Berührungen des Abdomens. $12^{\mathrm{h}}$ Nachts hatte es sich bereits etwas wiedererholt, $4^{\text {h }}$ Nachts frass es wieder

${ }^{1}$ Diese Zeitschrift. Bd. XVI. S. 268. 
und blieb von dann an gesund. Auf die starke Depression erfolgte nicht wie nach dem anfänglichen Abfall bei Injection kleinerer Dosen, eine Steigerung der Temperatur, sondern ein Uebergang zur Norm.

Dieses Thier hatte sich demgemäss von einer enorm hohen Dosis Gift erholt, während $1 / 25$ dieser Dosis von Cholerabacillen den sicheren Tod des. Thieres hätte herbeiführen müssen. Deshalb kann dieses Gift unmöglich das specifische Choleragift sein, sondern wir müssen Pfeiffer Recht geben und in ihm eins ron den von ihm als secundäre Gifte bezeichneten Stoffen suchen. Wir müssen ihm etwa die Stelle eines Adjuvans anweisen, da es doch sicher in alten Culturen nachgewiesen werden kann ohne chemische Eingriffe, und auch wohl in jungen vorhanden sein dürfte, obwohl man für diese immerhin den Einwurf machen dürfte, dass wir es durch unsere Manipulationen hier erst bilden.

Wichtiger und bedeutungsvoller als diese Frage erscheint uns der zweite Punkt, ob wir im Stande sind, durch Behandlung mit unserem Toxin eine Immunität gegen Cholera hervorzurufen.

Wiederholentlich haben wir Thiere mit dem aus den gekochten Bakterien hergestellten Filtrat vorbehandelt durch intraperitoneale wie subcutane Infection. Wir wandten dabei die verschiedensten Dosirungen an und wiederholten die Dosis mehrere Male hintereinander. Impften wir jedoch die Thiere mit Choleraculturen etwa 8 bis 10 Tage nach der letzten Infection mit Toxin, so gingen dieselben ausnahmslos zu Grunde. Auf Einzelheiten gehe ich hier nicht weiter ein. Es war nun möglich, dass die Thiere von dem in dem Filtrat enthaltenen wirksamen Gift nur eine zu geringe Dosis bekommen hatten und wurden demgemäss mit dem reineren Gift die Versuche wiederholt. Es schien uns vortheilhaft die Vorbehandlung des öfteren zu wiederholen, ehe wir zur Cholerainfection schritten.

Die erste Injection des in Wasser gelösten, mittels Alkohol gewonnenen Toxins zeigte folgende stündliche Curve:

$$
\begin{array}{lllll}
38 \cdot 0 & 38 \cdot 6 & 39.4 & 38.6 & 38 \cdot 3 .
\end{array}
$$
Stunden.

Das Thier, welches nicht sehr elend war, erholte sich nack einigen

Am folgenden Tage fand die zweite Injection statt; die stündliche Temperaturcurve war:

$$
\begin{array}{lllllll}
37 \cdot 9 & 37.2 & 36.9 & 38 \cdot 1 & 38.8 & 38.4 & 38 \cdot 0 .
\end{array}
$$

Tags darauf wurden dem Thiere eine halbe 24 Stunden bei $37^{\circ} \mathrm{C}$. gewachsene Agarcholerastrichcultur injicirt. Die Curve war stündlich gewessen :

$$
\begin{array}{llllll}
38.0 & 38 \cdot 0 & 39 \cdot 0 & 38.5 & 38.0 & 37 \cdot 5
\end{array}
$$

Das Thier erholte sich demgemäss von diesem Eingriff, und ist bis jetzt völlig gesund, während mehrere Controlthiere prompt eingingen. Dieser Versuch wurde wiederholt mit genauer Dosirung des Giftes. 
Ein Meerschweinchen von $280 \mathrm{grm}$ Gewicht erhält $0.006^{\mathrm{grm}}$ Toxin, also mehr als die maximal entsprechende tödtliche Dosis Cholerabacillen, vom Prodigiosus in $1 / 2{ }^{\mathrm{ccm}} \mathrm{H}_{2} \mathrm{O}$ aufgelöst intraperitoneal. Die Temperaturcurve war:

$\begin{array}{ccccccccc}10^{\mathrm{h}} & 10^{1} /^{\mathrm{h}} & 11^{\mathrm{h}} & \mathbf{1 2}^{\mathrm{h}} & 1^{\mathrm{h}} & 2^{\mathrm{h}} & 3^{\mathrm{h}} & 4^{1} /^{\mathrm{h}} & 6^{\mathrm{h}} \\ 38 \cdot 0 & 38 \cdot 9 & 39 \cdot 8 & 39 \cdot 3 & 39.2 & 39 \cdot 0 & 38 \cdot 8 & 38 \cdot 7 & 38 \cdot 3 .\end{array}$

Nach der Injection klagte das Thier etwas, war aber sonst nicht sehr krank. Am folgenden Tage erhält es $0.012^{\mathrm{grm}}$ Gift ebenfalls intraperitoneal in $1 / 4$ ecr $\mathrm{H}_{2} \mathrm{O}$ aufgelöst. Die Temperaturen waren:

$\begin{array}{cccccc}1^{1 / 2^{\mathrm{h}}} & 2^{\mathrm{h}} & 3^{\mathrm{h}} & 4^{1} / 2^{\mathrm{h}} & 6^{\mathrm{h}} & 8^{\mathrm{h}} \\ 38.1 & 38.9 & 38.8 & 39.5 & 39.2 & 38 \cdot 4 .\end{array}$

Tags darauf wurde dem Thier eine ganze, 24 Stunden alte, im Brütofen gewachsene, schräge Agarcholeracultur injicirt. Die Temperaturen waren:

$$
\begin{array}{cccccc}
1^{\mathrm{h}} & 2^{\mathrm{h}} & 3^{\mathrm{h}} & 5^{1} /^{\mathrm{h}} & 7^{1} / 2^{\mathrm{h}} & 9^{\mathrm{h}} \\
37 \cdot 0 & 37.0 & 37 \cdot 0 & 38 \cdot 0 & 38 \cdot 0 & 37 \cdot 5 .
\end{array}
$$

Ohne grosse Beschwerden hatte das Thier den schädigenden Eingriff überstanden, während das Controlthier unter dem typischen Bilde der Choleravergiftung erlag.

Issaeff ${ }^{1}$ hat nachgewiesen, dass auch durch Injectionen von Wasser, Nucleïnsäure, Tuberculin u. a. m. eine vorübergehende Immunität gegen eine nachfolgende Cholerainfection erreicht werden kann; ein Zustand, der im Wesentlichen auf der durch die Injectionen in den Bauch hervorgerufenen Lenkocytheneinwanderung und damit verbundenen Phagocythose beruht. Lässt man diesen Zustand vorübergehen, so sterben die Thiere genau so gut, wie die nicht vorbehandelten Controlthiere.

Wir werden sehen, dass diese Erklärung wohl für die Vorbehandlung mit Wasser, Nucleïnsäure, Tuberculin u. s. w. ausreicht, nicht aber für die mit Bakterien, z. B. dem Micrococcus prodigiosus. Erstens sind wir im Stande, durch intraperitoneale Injection von Prodigiosus und anderen Bakterienculturen ein Krankheitsbild genau wie nach der Injection mit Choleraculturen hervorzurufen, was wir durch Injection mit Wasser, Tubercalin wenigstens in den den Bacillen entsprechenden Dosen nicht vermögen, zweitens aber, und dieses scheint uns am bedeutungsvollsten, berichtet Klein über Versuche, in denen er durch vorhergehende wiederholte subcutane Injection der Meerschweinchen mit lebender oder sterilisirter Cultur des Spirillum Finckler, des Bacillus coli oder des Bacillus prodigiosus einen choleragiftfesten Zustand erzielte. Er verfügt über eine ganze Reihe von Meersehweinchen, die nach wiederholter subcutaner Injection sterilisirter Cultur der betreffenden Meerschweinchen und nachdem die Thiere sich wieder während mehrerer Tage von der hierdurch bedingten vorübergehenden Krankheit erholt hatten, hierauf mit letalen (am Controlthiere erprobten) Dosen der lebenden Choleracultur intraperitoneal

1 A. a. 0 . 
WEITERE MTTTHEILUNGEN ÜBER INTRAPERITONEALE INFECTION. 483

inficirt wurden, welche alle dieser Injection gegenüber sich als choleragiftfest zeigten. Ich habe ein Paar Meerschweinchen von 245 und $255^{\mathrm{grm}}$ mehrere Tage hintereinander 1 bis $2^{\mathrm{cem}}$ sterilisirtes Wasser subcutan injicirt und reagirten die Thiere auf Injection der grōsseren Dosen stets mit einem Temperaturanstieg. Als wir aber 24 Stunden nach der letzten Injection den Thieren eine intraperitoneale Injection von $3 / 4$ einer 24stündigen Agarcultur Cholerabacillen machten, erlagen die Thiere dieser Infection in gleicher Zeit und gleicher Weise wie nicht vorbehandelte Thiere.

Um so interessanter war es zu erfahren, ob die aus den Bakterien - wir nahmen den Micrococcus prodigiosus - dargestellten Toxine einen Impfschutz bei subcutaner Application gegen die nachfolgende Cholerainfection bewirkten.

Ein Meerschweinchen, $240 \mathrm{grm}$ schwer erhält $0.012^{\mathrm{grm}}$ Prodigiosustoxin in $1 / 2{ }^{\mathrm{cm}} \mathrm{H}_{2} \mathrm{O}$ aufgeschwemmt subcutan in die Nackengegend. Temperatur:

$$
\begin{array}{cccc}
1^{1} / 2^{\mathrm{h}} & 2^{\mathrm{h}} & \mathbf{3}^{\mathrm{h}} & 4^{1} / \mathbf{2}^{\mathrm{h}} \\
38 \cdot 3 & 38 \cdot 5 & \mathbf{3 8 . 4} & 38 \cdot 4 .
\end{array}
$$

Am folgenden Tage Injection von $0.01^{\mathrm{grm}}$ in $0.2^{\mathrm{cm}} \mathrm{H}_{2} \mathrm{O}$ in derselben Weise. Temperatur:

$$
\begin{array}{cccccc}
3^{\mathrm{h}} & 4^{\mathrm{h}} & 5^{\mathrm{h}} & 6^{\mathrm{h}} & 8^{\mathrm{h}} & 10^{\mathrm{h}} \\
38.4 & 38.6 & 39.5 & 39.0 & 39.0 & 38.3 .
\end{array}
$$

24 Stunden später erfolgte die Injection von einer Agarcultur 24 stün. diger Cholerabacillen intraperitoneal. Aber während das in gleicher Zeit mit derselben Giftdosis - bereits oben erwähnte - intraperitoneal geimpfte Thier die Infection überstand ohne Temperatursturz, ging dieses Thier ein wie das nicht rorbehandelte Controlthier. Die Obduction ergab den gewöhnlichen nach Cholerainfection beobachteten Befund, im Peritonealexsudat fanden sich mässig viele Bacillen.

Ein Impfschatz hatte somit in keiner Weise stattgefunden. Es fragte sich nun, wie verhielt es sich mit dem durch intraperitoneale Vorbehandlung erzielten Impfschutz. Beruhte derselbe etwa auch nur auf der Phagocytose? Aus der Zahl der mehrfach mit demselben übereinstimmenden Resultat ausgeführten Versuche führe ich nur zwei als Beispiele an.

Das bereits oben erwähnte Meerschweinchen, welches $0.3 \mathrm{grm}$ des Prodigiosustoxin bekommen hatte, wurde 6 Tage später mit einer Wasseraufschwemmung von $1^{\mathrm{eem}} 24$ stündiger Choleraagaragarcultur injicirt. Das Thier bot ein Krankheitsbild dar, genau wie das. nicht vorbehandelte Controlthier, und ging trotz der enormen Pyrotoxininjection, die etwa dem 25 fachen der absolut tödtlichen Cholerabacillenmenge entsprach, genau so prompt ein wie das Controlthier. Die Obduction ergab den gewöhnlichen Cholerabefund wie beim Controlthier; im reichlichen Exsudat fanden sich zahlreiche, lebhaft bewegliche Cholerabacillen in Reincultur. 
Ein anderes Thier von $225^{\mathrm{grm}}$ Gewicht erhält $0.024^{\mathrm{grm}}$ Toxin vom Prodigiosus intraperitoneal in $0.2^{\mathrm{cm}} \mathrm{H}_{2} \mathrm{O}$. Temperatur:

$\begin{array}{ccccccccc}10^{\mathrm{h}} & 10^{1 / 2^{\mathrm{h}}} & 11^{\mathrm{h}} & 12^{\mathrm{h}} & 1^{\mathrm{h}} & 3^{\mathrm{h}} & 4^{1 / 2^{\mathrm{h}}} & 6^{\mathrm{h}} & 8^{\mathrm{h}} \\ 38.3 & 38.2 & 39.2 & 39.8 & 39.6 & 39 \cdot 2 & 39.0 & 39.0 & 38.0\end{array}$

Am folgenden Tage erhält das Thier $0.04^{\mathrm{grm}}$ derselben Masse in $0.2^{\mathrm{ccm}} \mathrm{H}_{2} \mathrm{O}$. Temperatur:

$\begin{array}{cccc}5^{\mathrm{h}} & 6^{\mathrm{h}} & 8^{\mathrm{h}} & 10^{\mathrm{h}} \\ 38 \cdot 3 & 39 \cdot 3 & 39 \cdot 5 & 38 \cdot 5\end{array}$

7 Tage später erlag dieses Thier der intraperitonealen Injection einer Wasseraufschwemmung von einer 24stündigen Agarcholeracultur wie das Controlthier.

Das nämliche Resultat hatten Wiederbolungen dieser Versuchsreihe, so dass wir als Ergebniss dieser Versuche wohl mit Sicherheit behaupten können, dass das von uns dargestellte Toxin nicht der den Bakterien eigenthümliche specifische Giftstoff ist und wir sind daher in die Nothwendigkeit versetzt, die Angaben Pfejffer's als roll und ganz zu Recht bestehend anzuerkennen.

Bis jetzt sind alle Versuche, das Gift darzustellen, als gescheitert zu betrachten, ob es je gelingen wird, das specifische, jedenfalls sicher vorhandene Gift zu gewinnen, ist äusserst fraglich bei der ungemeinen Empfindlichkeit und leichten Zerstörbarkeit desselben.

Eine Einwirkung von $60^{\circ} \mathrm{C}$. nur wenige Stunden lang genügt schon, um das specifische Gift zu zerstören. Wir konnten durch Versuche eruiren, dass Bakterienmengen, welche 20 Minuten auf $60^{\circ} \mathrm{C}$. erhitzt, einen typischen Temperatursturz und Tod bewirkten, in gleicher Dosis nach mehrstündigem Erwärmen nur noch die Pyrotoxinwirkung erkennen liessen, so dass an Stelle des beobachteten Temperatursturzes eine vorübergehende Steigerung um 2 bis $3^{\circ} \mathrm{C}$. statthatte, welcher darauf die Norm folgte, so dass wir es völlig in der Hand haben, entweder einen Temperatursturz, bedingt durch das specifische Gift, oder eine Temperatursteigerung, bedingt durch das allgemeine Toxin mit unserer Bakterienmasse hervorzurufen.

Es ist das Verdienst ron R. Pfeiffer und Issaeff, in der dauernden Immunität gegenüber jeder nachfolgenden Cholerainfection nach einmal erfolgter Grundimmunisirung durch Cholerabacillen ein äusserst feines Reagens für die Diagnostik des Cholerabacillus gefunden zu haben.

Wir können diese Thatsache auf Grund eigener Versuche nur bestätigen. Da nach Angaben R. Pfeiffer's ${ }^{1}$ die echte Choleraimmunität der Meerschweinchen noch 3 bis 4 Monate nach der Immunisirung anhält,

A. a. 0 . 
Weitere MItThHeILUNGen ÜBer INTRaperitoneaLe INGeCTiON. 485

so erscheint es uns geboten - und Aufgabe grösserer Institute müsste es sein, diese Arbeit auszuführen -, stets eine grössere Anzahl Meerschweinchen gegen Cholera zu immunisiren. Findet man dann - wie in letzter Zeit sich die Befunde so ausserordentlich häufen - einen choleraähnlichen Vibrio, so haben wir ein mächtiges diagnostisches Hülfsmittel. Sterben die choleraimmunisirten Thiere, so hatten wir es nicht mit einem Cholerabacillus zu thun, bleiben sie am Leben, so muss man - wenigstens nach dem jetzigen Stande der ganzen Frage - die Diagnose auf Cholerabacillen stellen, mag auch immerhin die Indolbildung ausbleiben, oder das Wachsthum in irgend einem Nährboden etwas von der Norm abweichen. Derartige Beobachtungen sind mehrfach in letzterer Zeit gemacht, nur auf diesem Wege lässt sich oft die Entscheidung treffen. Wir halten für den concreten Fall unseren Vorschlag für sehr . viel besser als den umgekehrten Weg, da er innerhalb 24 Stunden gestattet, mit absoluter Sicherheit, besonders in den zweifelhaften Fällen, die Diagnose zu stellen. Man wird alsdann an Stelle der stets zweifelhaften intraperitonealen Infection lieber der subcutanen oder pleuralen Application den Vorzug geben und wenn irgend möglich, mehrere Thiere verwenden. Wir werden uns erlauben, in einer späteren Arbeit auf diesen Punkt noch einmal zurückzugreifen.

Danzig, den 10. April 1894. 